Selectively etching lanthanum to engineer surface cobalt enriched $\mathrm{LaCoO}_{3}$ perovskite catalysts for toluene combustion

Huawei Chen, Wei Cui, Dunfei Li, Qingqing Tian, Jianjun He, Qing Liu, Xian Chen, Mifen Cui, Xu Qiao*, Zhuxiu Zhang, Jihai Tang, and Zhaoyang Fei*

State Key Laboratory of Materials-Oriented Chemical Engineering, College of Chemical Engineering, Nanjing Tech University, Nanjing 211816, China

\title{
*Corresponding Authors
}

Tel.: +862583172298.Fax: +862583172298.E-mail:qct@njtech.edu.cn

Tel.: +8625 83587168.Fax: +862583587168.E-mail:zhaoyangfei@njtech.edu.cn 
Fig. S1 XRD patterns of LCO-OS and LCO-WI catalysts.

Fig. S2 (HR)TEM images of LCO-OS (a, c) and LCO-WI (b, d) catalysts.

Fig. S3 (a) $\mathrm{N}_{2}$ adsorption-desorption isotherms and (b) pore-size distributions of LCO-OS and LCO-WI catalysts.

Table S1 Detailed data of crystallite size $\left(\mathrm{d}_{\mathrm{XRD}}\right)$, specific surface area $(\mathrm{SSA})$, pore volume $\left(\mathrm{V}_{\mathrm{p}}\right)$ and pore size $\left(\mathrm{D}_{\mathrm{p}}\right)$ of LCO-OS and LCO-WI catalysts.

Fig. 44 Pore volume and pore size of all LCO catalysts with different $\mathrm{CoO}_{x}$ content.

Fig. $\mathbf{S 5} \mathrm{H}_{2}$-TPR curves of LCO-OS and LCO-WI catalysts.

Fig. $\mathbf{S 6} \mathrm{O}_{2}$-TPD curves of LCO-OS and LCO-WI catalysts.

Table $\mathbf{S 2} \mathrm{H}_{2}$ consumption and $\mathrm{O}_{2}$ desorption of LCO-OS and LCO-WI catalysts

Fig. S7 Relationship between the $\mathrm{H}_{2}$ consumption, the $\mathrm{O}_{2}$ desorption (Region II) and $\mathrm{CoO}_{x}$ content.

Fig. S8 Catalytic oxidation performances of toluene over LCO-OS and LCO-WI catalysts.

Fig. S9 XRD patterns of all used catalysts.

Table S3 Comparison of toluene catalytic oxidation over other catalysts reported in the literature. 


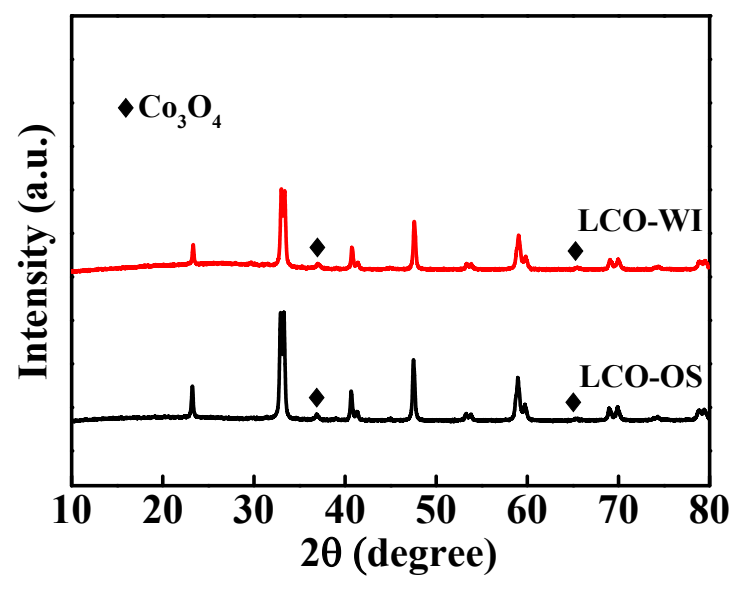

Fig. S1 XRD patterns of LCO-OS and LCO-WI catalysts. 


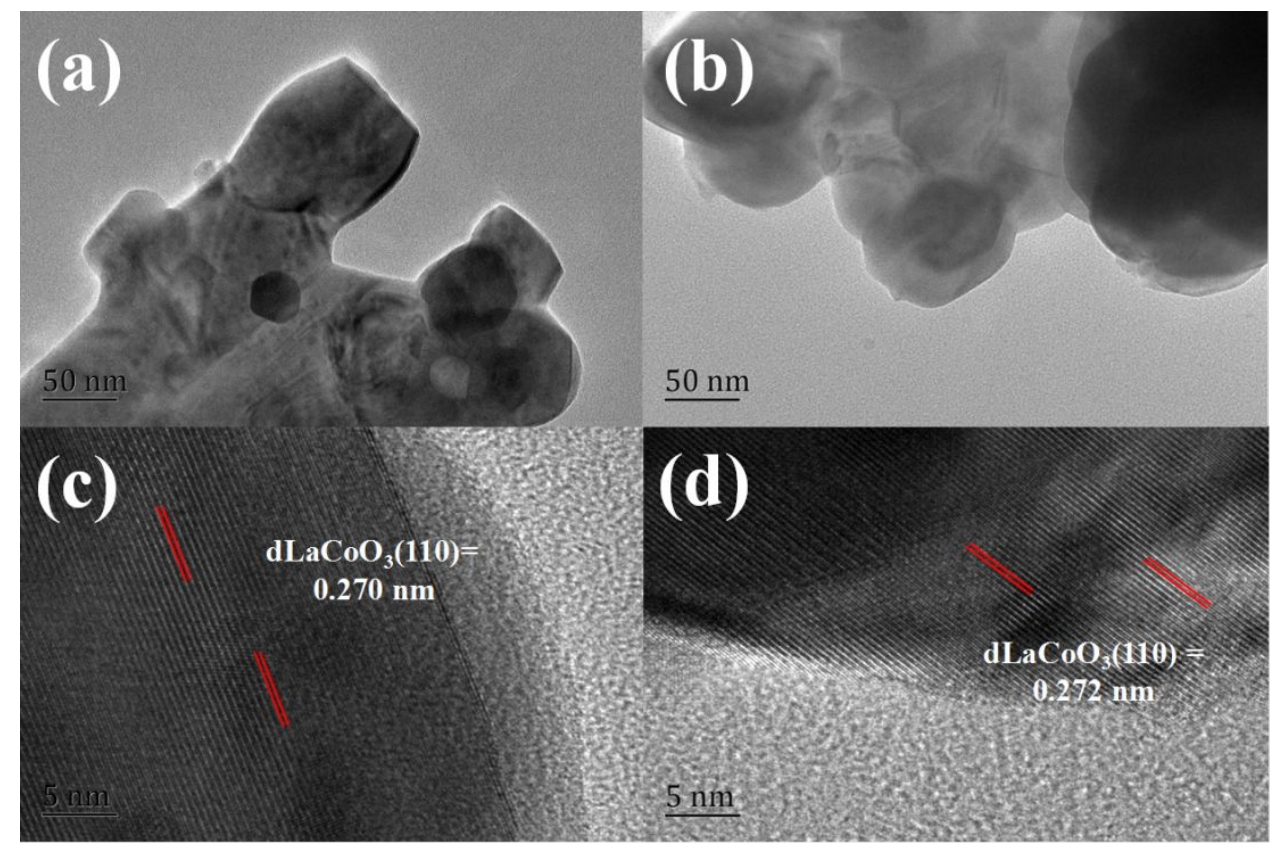

Fig. S2 (HR)TEM images of LCO-OS (a, c) and LCO-WI (b, d) catalysts. 

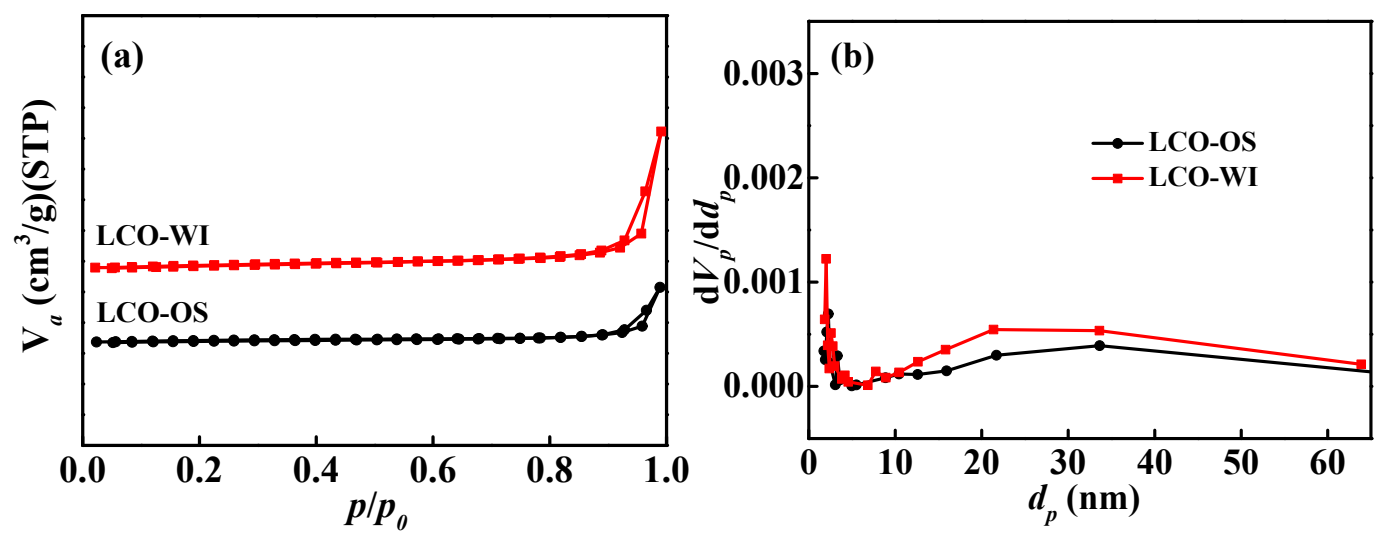

Fig. S3 (a) $\mathrm{N}_{2}$ adsorption-desorption isotherms and (b) pore-size distributions of LCO-OS and LCO-WI catalysts. 
Table S1 Detailed data of crystallite size $\left(\mathrm{d}_{\mathrm{XRD}}\right)$, specific surface area (SSA), pore volume $\left(\mathrm{V}_{\mathrm{p}}\right)$ and pore size $\left(\mathrm{D}_{\mathrm{p}}\right)$ of LCO-OS and LCO-WI catalysts

\begin{tabular}{lcccc}
\hline \multicolumn{1}{c}{ Catalysts } & $\mathrm{d}_{\mathrm{XRD}^{a}}(\mathrm{~nm})$ & $\begin{array}{c}\mathrm{SSA}^{b} \\
\left(\mathrm{~m}^{2} / \mathrm{g}\right)\end{array}$ & $\begin{array}{c}\mathrm{V}_{\mathrm{P}}{ }^{c} \\
\left(\mathrm{~cm}^{3} / \mathrm{g}\right)\end{array}$ & $\begin{array}{c}\mathrm{D}_{\mathrm{P}}{ }^{c} \\
(\mathrm{~nm})\end{array}$ \\
\hline LCO-OS & 16.2 & 6 & 0.03 & 3.3 \\
LCO-WS & 16.5 & 9 & 0.05 & 3.5 \\
\hline
\end{tabular}

${ }^{a}$ Calculated by Scherrer formula.

${ }^{b}$ Calculated by BET method.

${ }^{c}$ Obtained by BJH method. 


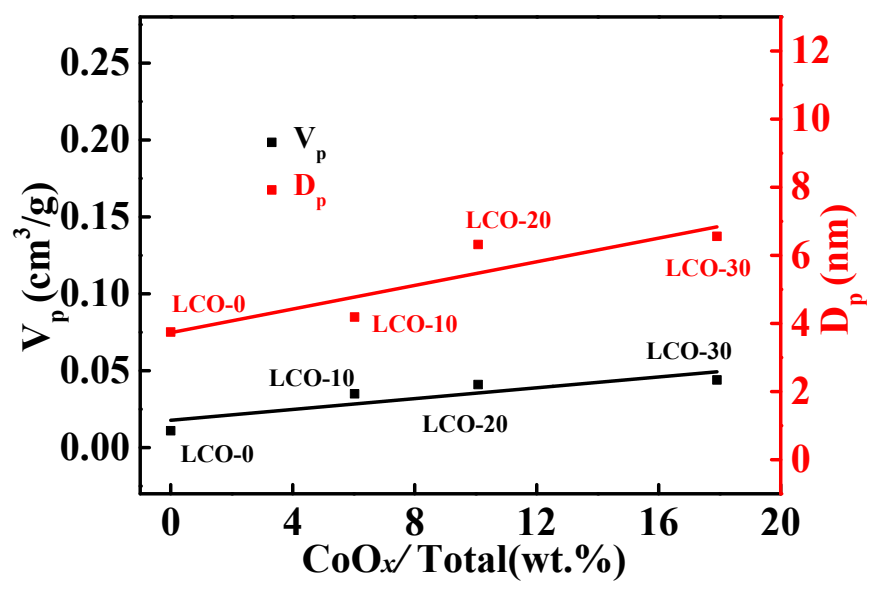

Fig. S4 Pore volume and pore size of all LCO catalysts with different $\mathrm{CoO}_{x}$ content. 


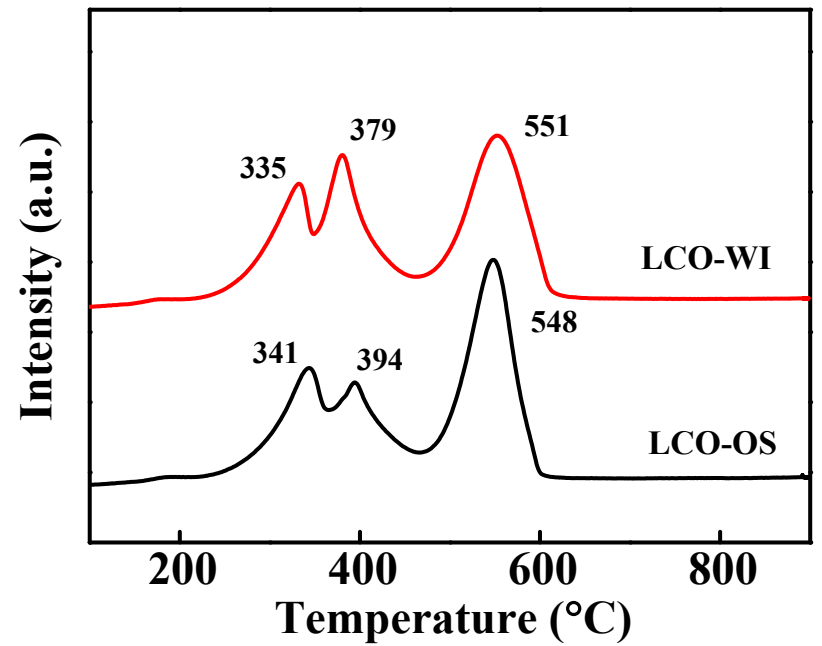

Fig. $\mathbf{S 5} \mathrm{H}_{2}$-TPR curves of LCO-OS and LCO-WI catalysts. 


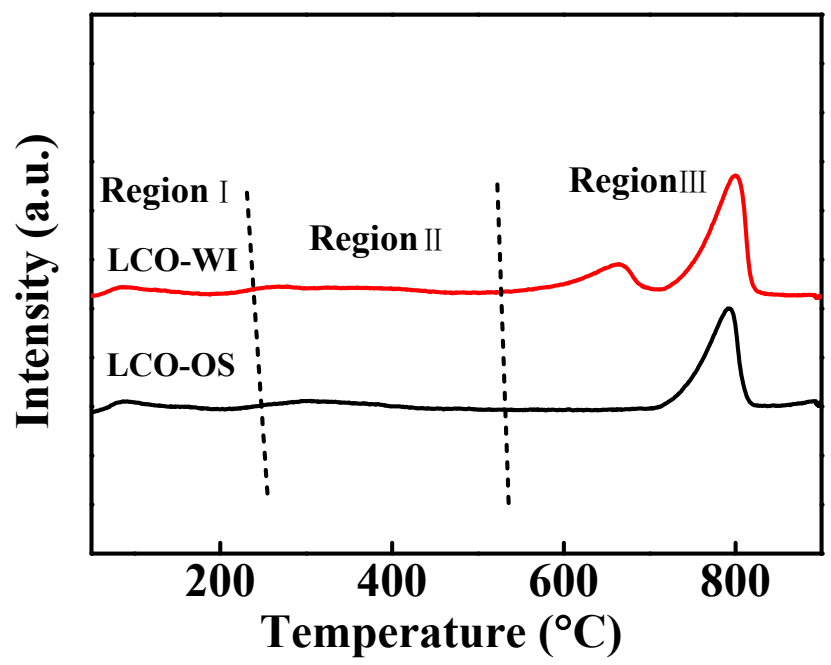

Fig. S6 $\mathrm{O}_{2}$-TPD curves of LCO-OS and LCO-WI catalysts. 
Table $\mathbf{S 2} \mathrm{H}_{2}$ consumption and $\mathrm{O}_{2}$ desorption of LCO-OS and LCO-WI catalysts

\begin{tabular}{|c|c|c|c|c|c|c|c|c|}
\hline \multirow[b]{2}{*}{ Catalysts } & \multicolumn{4}{|c|}{$\mathrm{H}_{2}$ consumption $\left(\mathrm{mmol} / \mathrm{g}_{\text {cat }}\right)$} & \multicolumn{4}{|c|}{$\mathrm{O}_{2}$ desorption $\left(\mathrm{mmol} / \mathrm{g}_{\mathrm{cat}}\right)$} \\
\hline & 1 st peak & 2nd peak & 3rd peak & Total & Region I & Region II & RegionIII & $\begin{array}{c}\text { Tota } \\
1\end{array}$ \\
\hline LCO-OS & 2.91 & 1.44 & 7.92 & 12.27 & 0.04 & 0.41 & 0.42 & 0.87 \\
\hline LCO-WI & 2.73 & 1.51 & 7.84 & 12.08 & 0.04 & 0.39 & 0.48 & 0.91 \\
\hline
\end{tabular}




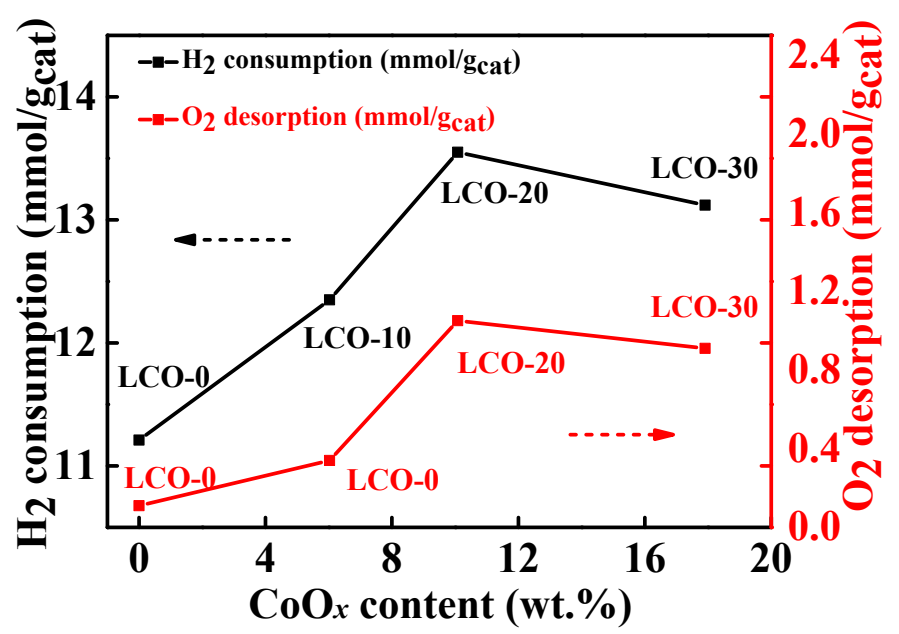

Fig. S7 Relationship between the $\mathrm{H}_{2}$ consumption, the $\mathrm{O}_{2}$ desorption (Region II) and $\mathrm{CoO}_{x}$ content. 


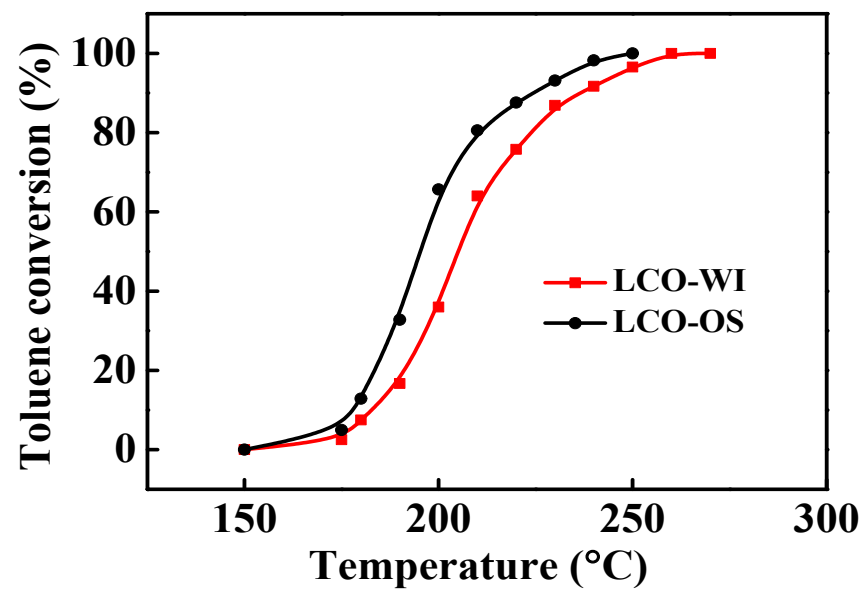

Fig. S8 Catalytic oxidation performances of toluene over LCO-WI and LCO-OS catalysts. 


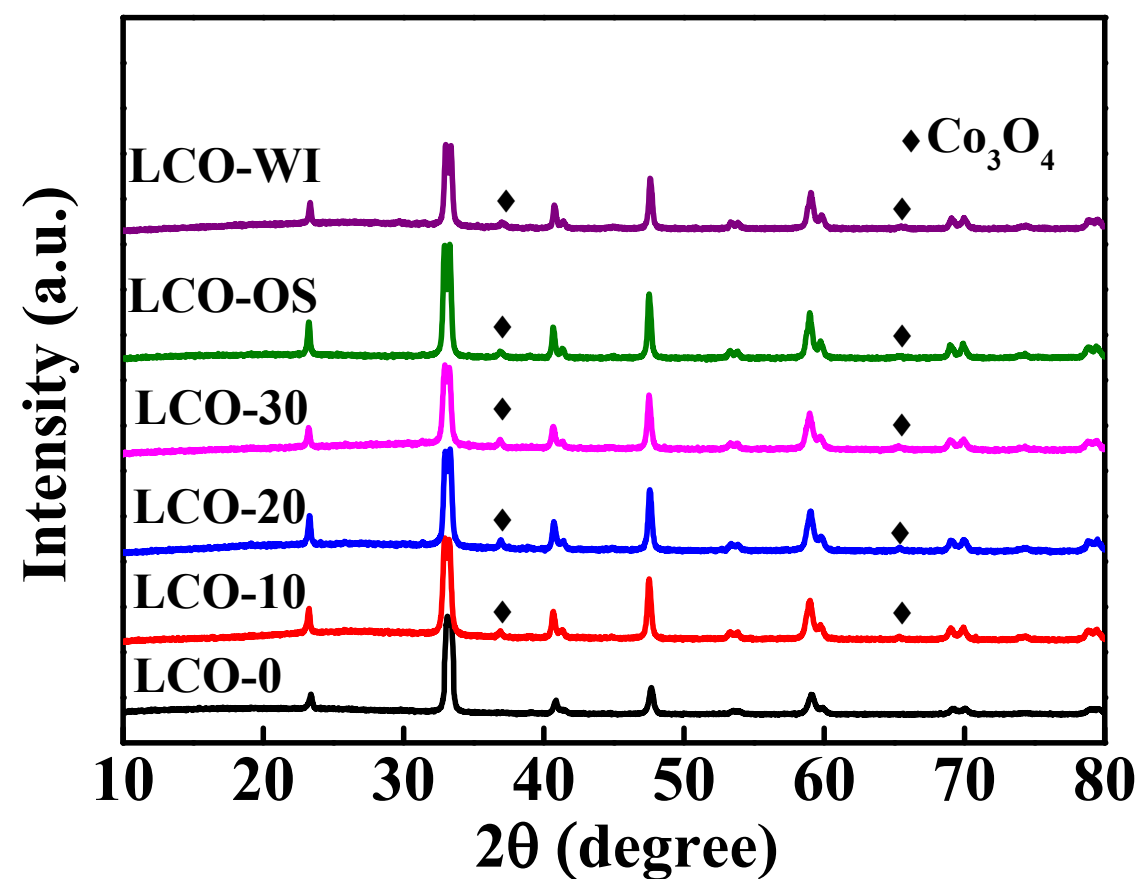

Fig. S9 XRD patterns of all used catalysts. 
Table S3 Comparison of toluene catalytic oxidation over other catalysts reported in the literature.

\begin{tabular}{|c|c|c|c|c|c|c|}
\hline \multirow{2}{*}{ Catalysts } & \multirow{2}{*}{ Reaction conditions } & \multicolumn{2}{|c|}{ Catalytic activities } & \multirow{2}{*}{$\begin{array}{l}\text { Reaction rate } \\
\times 10^{-8}\left(\mathrm{~mol} / \mathrm{g} \cdot \mathrm{s}^{-1}\right)^{a}\end{array}$} & \multirow{2}{*}{$\mathrm{Ea}(\mathrm{kJ} / \mathrm{mol})$} & \multirow{2}{*}{ Ref. } \\
\hline & & $\mathrm{T}_{50}\left({ }^{\circ} \mathrm{C}\right)$ & $\mathrm{T}_{90}\left({ }^{\circ} \mathrm{C}\right)$ & & & \\
\hline LCO-20 & $1000 \mathrm{ppm}, 15000 \mathrm{~h}^{-1}$ & 184 & 206 & 15.3 & 46 & This work \\
\hline $\mathrm{Co}_{3} \mathrm{O}_{4} / 3 \mathrm{DOM}$ LSCO & $1000 \mathrm{ppm}, 20000 \mathrm{~h}^{-1}$ & 210 & 227 & 7.4 & 43.3 & {$[1]$} \\
\hline $\mathrm{La}_{0.7} \mathrm{Sr}_{0.3} \mathrm{Co}_{0.8} \mathrm{Fe}_{0.2} \mathrm{O}_{3}$ & $500 \mathrm{ppm}, 5700 \mathrm{~h}^{-1}$ & 220 & 260 & 1.67 & 58.9 & {$[2]$} \\
\hline GLC-15 & $1000 \mathrm{ppm}, 30000 \mathrm{~h}^{-1}$ & 247 & 258 & 5.6 & 63.2 & [3] \\
\hline $\mathrm{Au} / 8 \mathrm{MnO}_{x} / 3 \mathrm{DOM}$ LSMO & $1000 \mathrm{ppm}, 20000 \mathrm{~h}^{-1}$ & 205 & 220 & 9.4 & 52.8 & {$[4]$} \\
\hline $\mathrm{MnO}_{x} / 3 \mathrm{DOM} \mathrm{LaMnO} 3$ & $1000 \mathrm{ppm}, 20000 \mathrm{~h}^{-1}$ & 193 & 215 & 19.8 & 48 & {$[5]$} \\
\hline $\mathrm{Ag} / \mathrm{LCO}-250$ & $1000 \mathrm{ppm}, 30000 \mathrm{~h}^{-1}$ & 231 & 239 & 3.7 & 57.5 & [6] \\
\hline
\end{tabular}

${ }^{a}$ calculated from the active test at $200{ }^{\circ} \mathrm{C}$.

[1] Li, X., Dai, H., Deng, J., Liu, Y., Zhao, Z., Wang, Y., Yang, H., Au, C.T. In situ PMMA-templating preparation and excellent catalytic performance of $\mathrm{Co}_{3} \mathrm{O}_{4} / 3 \mathrm{DOM} \mathrm{La}_{0.6} \mathrm{Sr}_{0.4} \mathrm{CoO}_{3}$ for toluene combustion. Appl. Catal., A 2013, 458, 11-20.

[2] Rousseau, S., Loridant, S., Delichere, P., Boreave, A., Deloume, J.P., Vernoux, P. $\mathrm{La}_{(1-\mathrm{x})} \mathrm{Sr}_{\mathrm{x}} \mathrm{Co}_{1-\mathrm{y}} \mathrm{Fe}_{\mathrm{y}} \mathrm{O}_{3}$ perovskites prepared by sol-gel method: Characterization and relationships with catalytic properties for total oxidation of toluene. Appl. Catal., B 2009, 88, 438-447.

[3] Yang, J., Li, L., Yang, X., Song, S., Li, J., Jing, F., Chu, W. Enhanced catalytic performances of in situ-assembled $\mathrm{LaMnO}_{3} / \delta-\mathrm{MnO}_{2}$ hetero-structures for toluene combustion. Catal. Today. 2019, 327, 19-27.

[4] Jiang, Y., Deng, J., Xie, S., Yang, H., Dai, H. Au/MnO ${ }_{x} / 3 \mathrm{DOM} \mathrm{La}_{0.6} \mathrm{Sr}_{0.4} \mathrm{MnO}_{3}$ : Highly Active Nanocatalysts for the Complete Oxidation of Toluene. Ind. Eng. Chem. Res. 2015, 54, 900-910.

[5] Liu, Y.; Dai, H.; Deng, J.; Du, Y.; Li, X.; Zhao, Z.; Wang, Y.; Gao, B.; Yang, H.; Guo, G. In situ poly(methyl methacrylate)-templating generation and excellent catalytic performance of $\mathrm{MnO}_{x} / 3 \mathrm{DOM}$ $\mathrm{LaMnO}_{3}$ for the combustion of toluene and methanol. Appl. Catal., B 2013, 140-141, 493-505.

[6] Chen, H., Wei, G., Liang, X., Liu, P., He, H., Xi, Y., Zhu, J. The distinct effects of substitution and deposition of Ag in perovskite $\mathrm{LaCoO}_{3}$ on the thermally catalytic oxidation of toluene. Appl. Surf. Sci. 2019, 489, 905-912. 